\title{
PROJETO DE UMA PASSARELA DE ESTRUTURA METÁLICA EM CAÇADOR-SC
}

Felipe Augusto Kemp ${ }^{1}$

Luiz Fernando Gardini²

\section{RESUMO}

Diversos métodos de transposição de objetos e cursos d'água foram adaptados durante o tempo, as pontes e passarelas foram as que sofreram as melhorias mais drásticas. Além do desenvolvimento de novos métodos de distribuir as cargas, foram descobertos novos materiais (como as estruturas de aço) para comporem as estruturas de fixação, recepção e transmissão dos esforços solicitantes. Toda essa adaptação se faz presente hoje nos projetos de novas pontes e passarelas, inclusive a passarela pressuposto deste trabalho. Este artigo se trata de um projeto no município de Caçador, Santa Catarina, onde os moradores dos bairros Santa Catarina, Krüger e DER, tendo em vista sua dificuldade de transpor o Rio do Peixe, manifestaram seu interesse na proposta de uma nova passarela localizada próximo ao Parque Central José Rossi Adami, fornecendo assim uma nova trajetória para os pedestres (especialmente cadeirantes) e encurtando consideravelmente a distância de seus percursos.

Palavras-Chave: Passarela; dimensionamento; estrutura metálica; Caçador.

\section{ABSTRACT}

Several implementation methods of objects and waterways transposing have been adapted over time, the bridges and footbridges were the ones that suffered the most drastic improvements. Besides the development of new loads distributing methods, new materials also have been discovered (such as steel structures) to compose the fixing structure, internal reception and transmission forces. All this adaptation is present today in the projects of new bridges and walkways, including the walkway assumption of this work. This article is about a project in the city of Caçador, Santa Catarina, where residents of the neighborhoods Santa Catarina, Krüger and DER, given its difficulty to cross the Rio do Peixe, expressed interest in the proposal of a new walkway located next the Central Park José Rossi Adami, thus providing a new

\footnotetext{
${ }^{1}$ Acadêmico do Curso de Engenharia Civil da Universidade Alto Vale do Rio do Peixe.

2 Professor Orientador. Graduado em Engenharia de Produção Mecânica pela Universidade do Oeste de Santa Catarina, Pós-Graduado em Engenharia de Segurança do Trabalho pela Universidade do Oeste de Santa Catarina e docente do Curso de Engenharia Civil pela Universidade Alto Vale do Rio do Peixe.
}

Ignis | Caçador | v.6 | n.2 | p. 64-84 | maio/ago. 2017 
path for pedestrians (especially wheelchair users) and considerably shortening the distance of their routes.

Keywords: Footbridge; scaling; metallic structure; Caçador.

\section{INTRODUÇÃO}

Diante das dificuldades enfrentadas por moradores dos bairros Santa Catarina, Krüger e DER em atravessar o Rio do Peixe, na cidade de Caçador-SC, foi solicitado à Prefeitura Municipal de Caçador (PMC) que projetasse uma travessia para pedestres, facilitando o acesso ao Parque Central José Rossi Adami, bem como ao bairro Centro. Apesar da PMC ter realizado um estudo para implantação desta travessia em 2009, ela não foi executada por escassez de verba, segundo funcionários do Instituto de Pesquisa e Planejamento Urbano de Caçador (IPPUC). Através deste trabalho, vamos propor uma segunda alternativa à $\mathrm{PMC}$, buscando solucionar este problema de mobilidade com soluções mais economicamente eficientes. Como finalidade deste trabalho, iremos detalhar as características que fazem do aço um dos melhores materiais para realização de uma passarela, bem como dimensionar uma passarela a ser localizada numa cidade do oeste catarinense.

Portanto, o objetivo geral deste artigo é realizar o projeto de implementação de uma passarela em estrutura metálica na cidade de Caçador-SC, a fim de promover maior facilidade de locomoção de pedestres (especialmente PNEs) dos bairros Santa Catarina, Krüger e DER para o bairro Centro e Parque Central José Rossi Adami.

Logo, os objetivos específicos são:

a) Definir o tipo de aço à ser utilizado, verificando suas propriedades e como poderá ser aplicado;

b) Definir a metodologia de cálculo e as normas à serem utilizada na passarela;

c) Dimensionar uma passarela em estrutura metálica. 


\section{FUNDAMENTAÇÃO TEÓRICA}

\section{PASASRELAS}

Segundo Fialho (2004), as passarelas podem ser analisadas e caracterizadas basicamente por quatro elementos: Tipo de acesso, tipo de tabuleiro, tipo de vedações e tipo de sistema estrutural utilizado.

Fialho (2004) ainda afirma que os acessos podem ser feitos em três situações de nível:

a) Duas extremidades nos mesmos níveis dos caminhamentos dos pedestres;

b) Apenas uma extremidade no mesmo nível do caminhamento do pedestre;

c) Duas extremidades em níveis diferentes dos caminhamentos dos pedestres.

Quanto ao tabuleiro, Pfeil (1983, p. 18) determina que "se denomina estrado ou tabuleiro de uma ponte a parte que serve de apoio imediato dos elementos de função viária, tais como vias férreas, pistas, passeio, dutos, etc."

Quadros (2012) complementa que a função do tabuleiro é receber diretamente as cargas que transitam pela ponte.

Quanto aps sistemas estruturais, podemos dizer que são definidores das principais características das passarelas. Podem definir sua forma e expressão plástica e impõem requisitos relativos a vãos, apoios, acessos, gabaritos e processos de montagem. Os vários tipos de passarelas podem ser classificados pelo material empregado (aço, concreto ou madeira), pela dimensão de seu vão (pequeno médio ou grande) ou pelo sistema estrutural utilizado (viga, treliça, arco, suspensão ou cabo estaiada) (FIALHO, 2004).

Um dos sistemas estruturais mais utilizados é o de vigas. As estruturas em vigas trabalham basicamente com esforços de cisalhamento e momento fletor (PFEIL, 1983).

Fialho (2004) mostra que a configuração física das vigas é das mais simples, basicamente composta por um único elemento apoiado ou engastado, em colunas ou apoios no solo. 


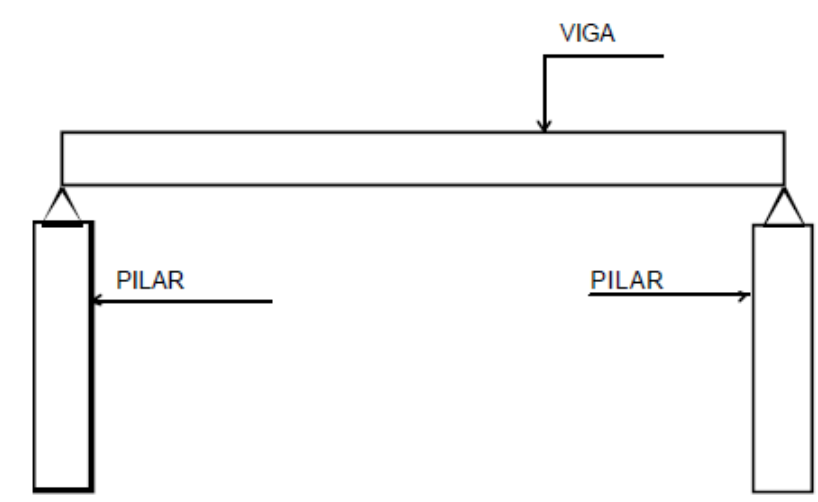

Figura 1 - Sistema estrutural de vigas

Fonte: Fialho (1998)

O autor explica que o sistema de vigas é o mais eficiente e barato para pequenos vãos (10 a 25 metros), tendo baixo custo de fabricação, pintura e manutenção, além de serem de fácil montagem, demandando utilização de equipamentos leves e pouca interferência no tráfego local.

ASTM-A36

Dentre os aços estruturais existentes atualmente, o mais utilizado e conhecido é o ASTM-A36, que é classificado como um aço carbono de média resistência mecânica (CBCA, 2014).

Apresentando uma boa soldabilidade, o aço A36 possui pequenas quantidades de carbono. Sendo composto principalmente (99\%) de ferro, sua resistência e usinabilidade são baixas. Possui uma superfície rugosa, sua resistência em relação a tração se encontra entre 58.000 psi e 79.800 psi, trabalhando com um máximo de elasticidade de 36.000 psi, sendo recomendado pela norma não ultrapassar um alongamento de $20 \%$ de sua seção (TRIMETAIS, 2015).

O projeto a ser desenvolvido, será dimensionado utilizando-se o açocarbono ASTM-A36, por ser mais comum e melhor adaptável no projeto em que será aplicado.

\section{METODOLOGIA E MATERIAL}

A logística de mobilidade até hoje é um fator preocupante dentro da 
estrutura organizacional de uma cidade, pois representa os meios de locomoção de pessoas e tráfego na mesma. Podem existir diversos obstáculos à serem atravessados quando se deseja partir de um ponto A para um ponto B, e este fator se majora quando o obstáculo à ser atravessado é um rio cruzando a cidade. Existem, atualmente, duas travessias próximas ao objeto de projeto deste trabalho, que são utilizadas para travessia do Rio do Peixe atualmente.

São elas as pontes da rodoviária e ponte do trabalhador, como podem ser observadas, respectivamente, nas figuras 8 e 9 :

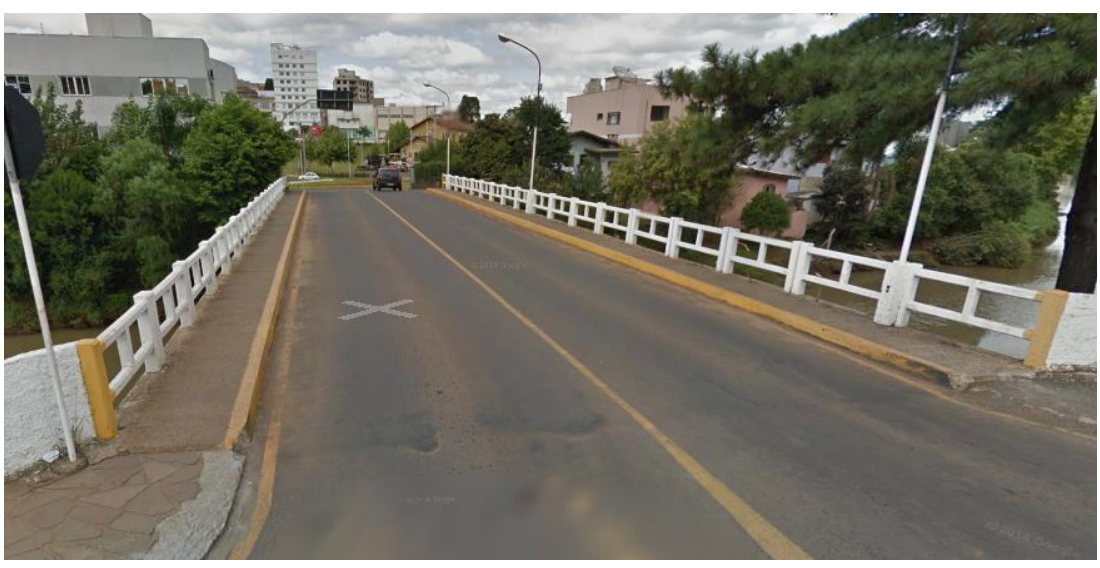

Figura 2 - Ponte da rodoviária

Fonte: O próprio autor (2017)

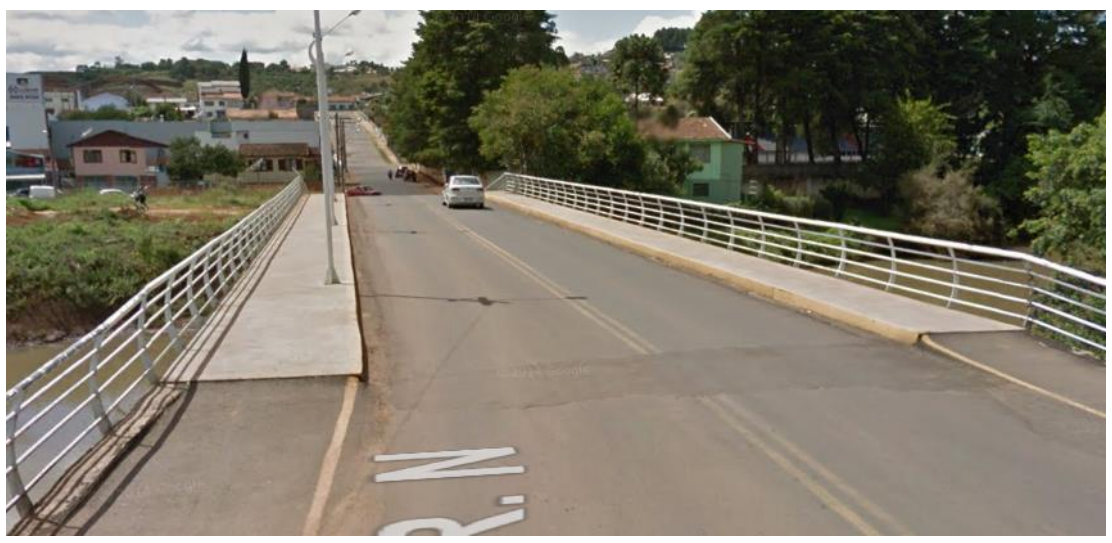

Figura 3 - Ponte do trabalhador

Fonte: O próprio autor (2017)

As dificuldades a serem solucionadas neste artigo refletem-se principalmente em dois pontos: 

a) Falta de acessibilidade e largura de paralelepípedo inferior à mínima na ponte da rodoviária;
b) Distância de $1 \mathrm{~km}$ entre as duas pontes, sem alternativas de travessias mais próximas.

A figura 4 a seguir detalha esta distância entre as travessias existentes, bem como a localização dos bairros:

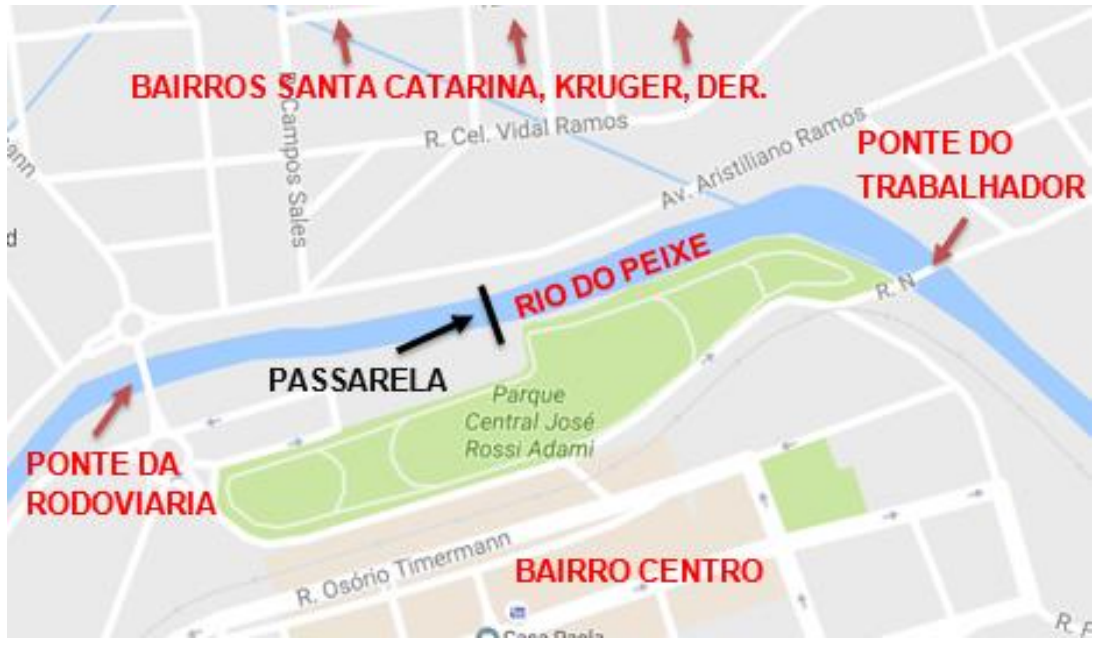

Figura 4 - Mapa de travessias existentes

Fonte: O próprio autor (2017)

Dentre as normas a serem utilizadas no projeto estrutural metálico, as principais são definidas por Gaspar (2008):

a) NBR 8800 (ABNT, 1986) - Projeto e execução de estruturas de aço de edifícios: método dos estados limites.

b) ASTM - American Society for Testing and Materials: especificações para fabricação do aço, acabamento dos perfis, etc.

c) AISC - American Institute of Steel Construction: especificações para projetos de prédios industriais ou residenciais em estruturas metálicas.

d) AASHO - American Assiciation of State Highway Officials: especificações para projeto de pontes rodoviárias metálicas.

e) NBR 6123 (ABNT, 1988) - Forças devidas ao vento em edificações.

f) NBR 6120 (ABNT, 1980) - Cargas para o cálculo de estruturas de edificações.

g) NBR 9763 (ABNT, 1987) - Aços para perfis laminados, chapas grossas e 
barras, usados em estruturas fixas.

h) NBR 7188 (ABNT, 1984) - Carga móvel em ponte rodoviária e passarela de pedestre.

A metodologia de cálculo a ser utilizada foi adaptada de Neto (2008), de acordo com a NBR 8800 (ABNT, 1986).

\section{APRESENTAÇÃO, ANÁLISE DOS DADOS E RESULTADOS RESULTADOS E DISCUSSÃO}

\section{CÁLCULO DA LARGURA DA PASSARELA}

Dimensionaremos a largura da passarela através da fórmula:

$B=\frac{Q}{d \times v}$

Onde:

$\mathrm{B}=$ Largura do tabuleiro em metros;

$Q=$ Número de pedestres atravessando a passarela por hora (estipulado em 300);

$\mathrm{d}=$ Densidade demográfica $\left(71,89 \mathrm{hab} / \mathrm{m}^{2}\right.$, segundo IBGE 2010)

$v=$ Velocidade $($ normal $=1,0 \mathrm{~m} / \mathrm{s}$, em horários de maior movimento $=$ $1,5 \mathrm{~m} / \mathrm{s})$.

Desta forma:

$$
B=\frac{300}{71,89 \times 1,5}
$$

$B \cong 3,00 \mathrm{~m}$ 


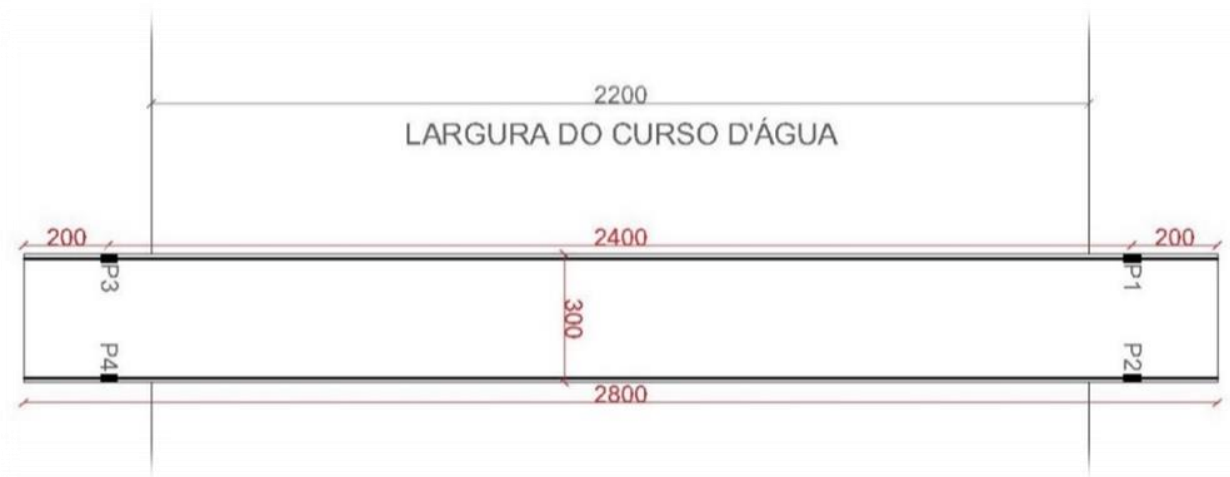

Figura 5 - Dimensões da passarela

Fonte: O próprio autor (2017)

\section{DEFINIÇÃO DAS CARGAS}

Assim como todas as estruturas de concreto armado, as de estruturas metálicas também possuem critérios para a definição de suas cargas, que podem ser divididas em dois grandes grupos: Cargas permanentes; e cargas variáveis.

O peso próprio consiste nos valores de carga que são transmitidos do próprio peso da estrutura para a estrutura em si, através da ação da gravidade. Ela pode variar de acordo com o tamanho da estrutura, material utilizado e altitude da localização.

Para cálculo do peso próprio dos materiais que compõem a passarela, precisamos dos pesos específicos de cada material. Vamos utilizar para vidro 26 $\mathrm{kN} / \mathrm{m}^{3}$, e para aço, conforme visto anteriormente, $78,5 \mathrm{kN} / \mathrm{m}^{3}$, de acordo com a NBR 6120/80 (ABNT, 1980).

As cargas totais sobre a viga que vamos dimensionar serão:

$$
P T=G+Q+C V
$$

Onde:

$G=$ Carga permanente total;

$Q=$ Carga acidental total;

$C V=$ Carga de vento total; 


\section{CARGAS PERMANENTES}

As cargas permanentes são aquelas que efetivamente agirão sobre a estrutura independente de tempo. A principal carga permanente que será utilizada nos cálculos é a carga de peso próprio.

O peso próprio total calculado sobre a viga que dimensionaremos será:

$$
G=P_{L}+P_{V}+P_{P}+P_{I}
$$

Onde:

$G=$ Carga permanente total sobre a viga;

$P_{L}=$ Peso da laje steeldeck sobre cada viga;

$P_{V}=$ Peso do vidro sobre cada viga;

$P_{P}=$ Peso dos pilares contendo a cobertura sobre cada viga;

$P_{I}=$ Peso próprio das vigas;

\section{PESO DA LAJE STEELDECK}

O peso da laje sobre as vigas será de:

$$
P_{L}=L_{L} \times L_{C} \times L_{H} \times L_{P}
$$

Onde:

$L_{L}=$ Largura da laje

$L_{C}=$ Comprimento da laje;

$L_{H}=$ Altura da laje;

$L_{P}=$ Peso específico da laje $\left(78,50 \mathrm{kN} / \mathrm{m}^{3}\right)$.

Portanto:

$P_{L}=3 \times 24 \times 0,0075 \times 78,50$ 


$$
P_{L}=42,39 k N
$$

Como a laje será bi-apoiada lateralmente sobre as duas vigas, seu peso próprio será posteriormente dividido por dois.

$$
\begin{aligned}
& P_{L}=\frac{42,39}{2} \\
& P_{L}=21,19 k N
\end{aligned}
$$

E por fim, para transformarmos numa carga distribuída sobre cada viga, dividiremos pelo comprimento da viga:

$$
\begin{aligned}
& P_{L}=\frac{21,19}{24} \\
& P_{L} \cong 0,9 \mathrm{kN} / \mathrm{m}
\end{aligned}
$$

PESO DOS VIDROS

O peso dos vidros sobre cada viga será calculado de forma que:

$$
P_{V}=V_{L} \times V_{C} \times V_{H} \times V_{P}
$$

Onde:

$V_{L}=$ Largura do vidro;

$V_{C}=$ Comprimento do vidro;

$V_{H}=$ Altura do vidro;

$V_{P}=$ Peso específico do vidro $\left(2500 \mathrm{kN} / \mathrm{m}^{3}\right)$.

Portanto:

$$
\begin{aligned}
& P_{V}=0,01 \times 1,5 \times 24 \times 26 \\
& P_{V}=9,36 k N
\end{aligned}
$$


Novamente, dividimos a carga encontrada pelo comprimento da viga, para transformarmos em uma carga distribuída:

$$
\begin{aligned}
& P_{V}=\frac{9,36}{24} \\
& P_{V} \cong 0,4 \mathrm{kN} / \mathrm{m}
\end{aligned}
$$

\section{PESO DOS PILARES E COBERTURA}

Para cálculo do peso da cobertura, vamos adaptar um conceito de NETO (2007, pg. 10), admitindo $0,15 \mathrm{kN} / \mathrm{m}^{2}$ para coberturas de estruturas leves, resultando em aproximadamente $8,4 \mathrm{kN}$ o peso total da cobertura, e 2,1 kN de carga concentrada sobre cada um dos quatro pilares.

Já para os pilares, vamos admitir um perfil qualquer, e calcular o peso próprio deste, alterando novamente posteriormente caso haja necessidade.

O pilar adotado para efeito de cálculo é um perfil soldado A36 da Açominas, com dimensões informadas na imagem à segur:

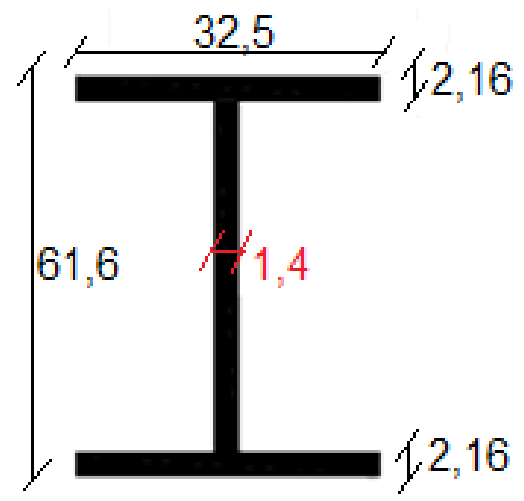

Figura 6 - Perfil admitido para o pilar Fonte: O próprio autor (2017)

Seu peso total será o peso próprio, mais o peso da cobertura:

$$
P_{P}=P I_{A} \times P I_{H} \times P I_{P E}+P I_{C}
$$


Onde:

$P I_{A}=$ Área do perfil;

$P I_{H}=$ Altura do perfil;

$P I_{P E}=$ Peso específico do perfil $\left(78,50 \mathrm{kN} / \mathrm{m}^{3}\right)$;

$P I_{C}=$ Peso da cobertura.

Portanto:

$P_{P}=0,022059 \times 2,6 \times 78,50+2,1$

$P_{P}=6,60 k N$

\section{PESO PRÓPRIO DAS VIGAS}

Para cálculo do peso próprio das vigas, vamos admitir também uma viga qualquer, e dimensiona-la para este peso próprio, alterando posteriormente caso haja necessidade. Para as vigas, vamos admitir o seguinte perfil:

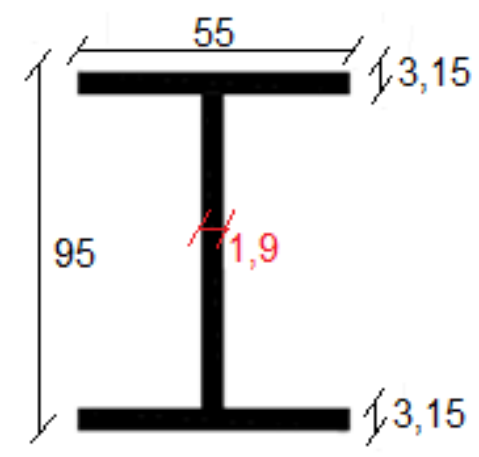

Figura 7 - Perfil admitido para a viga

Fonte: O próprio autor (2017)

Tabela 8 - Detalhamento do perfil de viga

\begin{tabular}{lllllllll}
\hline \multicolumn{1}{l}{ DIMENSÕES } & \multicolumn{10}{c}{} & EIXO X-X \\
\hline perfil & $\mathrm{d}(\mathrm{cm})$ & $\mathrm{bf}(\mathrm{cm})$ & $\mathrm{tf}(\mathrm{cm})$ & $\mathrm{tw}(\mathrm{cm})$ & $\mathrm{h}(\mathrm{cm})$ & $\mathrm{A}\left(\mathrm{cm}^{2}\right)$ & $\mathrm{Ix}\left(\mathbf{c m}^{4}\right)$ & $\mathrm{Wx}\left(\mathbf{c m}^{3}\right)$ \\
\hline $950 \times 404$ & 95 & 55 & 3,15 & 1,9 & 88,7 & 515 & 841600 & 17720 \\
\hline
\end{tabular}


Fonte: Neto (2008)

Seu peso próprio por metro será:

$P_{I}=I_{A} \times I_{P}$

Onde:

$I_{A}=$ Área do perfils;

$I_{P}=$ Peso específico do perfil $\left(78,50 \mathrm{kN} / \mathrm{m}^{3}\right)$;

Portanto:

$P_{I}=0,0515 \times 78,50$

$P_{I}=4,05 \mathrm{kN} / \mathrm{m}$

Desta forma, teremos que a carga permanente linear total sobre cada viga será:

$$
\begin{aligned}
& G=4,05+0,40+0,9 \\
& G=13,445 \mathrm{kN} / \mathrm{m}
\end{aligned}
$$

CARGAS VARIÁVEIS

\section{Cargas de pedestres}

De acordo com a NBR 7188/82, para passarelas devemos considerar 5 $\mathrm{kN} / \mathrm{m}^{2}$ a carga móvel de pedestres.

Para transformar esta carga em unidade linear, vamos multiplica-la pela área da laje (onde a carga de pedestres será aplicada): 


$$
A_{P}=T_{L} \times T_{C} \times 5 k N / m^{2}
$$

Onde:

$T_{L}=$ Largura do tabuleiro;

$T_{C}=$ Comprimento livre do tabuleiro (entre pilares);

Portanto:

$$
\begin{aligned}
& A_{P}=3 \times 24 \times 5 \\
& A_{P}=360 \mathrm{kN}
\end{aligned}
$$

Dividimos a carga por dois, visto que a laje será apoiada em duas vigas uniformes:

$$
A_{P}=180 \mathrm{kN}
$$

Por fim, dividimos pelo comprimento útil da viga, para obtermos uma carga distribuída:

$$
\begin{aligned}
& A_{P}=\frac{180}{24} \\
& A_{P}=7,5 \mathrm{kN} / \mathrm{m}
\end{aligned}
$$

Como esta será a única carga acidental atuante, temos que:

$$
Q=7,5 \mathrm{kN} / \mathrm{m}
$$

\section{Vento}

Todo o cálculo de vento está de acordo com a NBR 6123/88 (ABNT), as 
seguintes equações foram utilizadas:

$$
\Delta_{P}=C_{p e} \times C_{p i} \times q
$$

Onde:

$\Delta_{P}=$ Diferença de pressão nas faces opostas (externa e interna);

$C_{p e}=$ Coeficiente de pressão externo (Adotado -1,0 por efeito venturi moderado);

$C_{p i}=$ Coeficiente de pressão interno (Adotado $-0,2$ por haverem 4 faces permeáveis);

$q$ = Pressão dinâmica do vento;

$$
q=0,613 \times V_{k}^{2}
$$

Onde:

$V_{k}=$ Velocidade característica do vento.

$$
V_{k}=V_{0} \times S_{1} \times S_{2} \times S_{3}
$$

Onde:

$V_{0}=$ Velocidade básica do vento ( $45 \mathrm{~m} / \mathrm{s}$ segundo mapa de isopetas);

$S_{1}=$ Fator topográfico (Considerado 1,0, por se tratar de terreno plano ou fracamente acidentado);

$S_{2}=$ Fator de rugosidade do terreno (Considerado 0,86, por se enquadrar na categoria III, Classe B, para altura $<=5 \mathrm{~m}$ );

$S_{3}=$ Fator estatístico (Considerado 0,88, por se encaixar no grupo 4).

Portanto:

$$
V_{k}=45 \times 1,0 \times 0,86 \times 0,88
$$




$$
\begin{aligned}
& V_{k}=34,056 \mathrm{~m} / \mathrm{s} \\
& q=0,613 \times(34,056)^{2} \\
& q=710,96 \mathrm{~N} / \mathrm{m}^{2} \\
& \Delta_{P}=(-1,0) \times(-0,2) \times 710,96 \\
& \Delta_{P}=142,192 \mathrm{~N} / \mathrm{m}^{2} \\
& \Delta_{P}=14,3 \mathrm{kN} / \mathrm{m}^{2}
\end{aligned}
$$

Como o valor obtido acima refere-se à uma rajada de vento em qualquer face da passarela, vamos considerar o pior caso sendo o vento atingindo a cobertura no sentido vertical, e dividimos seu valor pelo comprimento útil da passarela para obter uma carga linear:

$$
\Delta_{P}=\frac{14,3}{24}=0,595 \mathrm{kN} / \mathrm{m}
$$

Desta forma, temos que:

$$
C V=0,595 \mathrm{kN} / \mathrm{m}
$$

Temos então que o peso total sobre cada viga será:

$$
\begin{aligned}
& P T=G+Q+C V \\
& P T=4,872+7,50+0,595 \\
& P T=12,967 \mathrm{kN} / \mathrm{m}
\end{aligned}
$$

\section{DIMENSIONAMENTO}

Conforme citado no item 2.2.1, o dimensionamento terá como base as fórmulas adaptadas de NETO (2008), de acordo com a NBR 8800 (ABNT, 1986). 


\section{Vigas}

Tipo de seção na flambagem lateral

Testamos a equação para duas situações para descobrir se a mesa é compacta ou não compacta:

$$
\frac{b}{t_{f}}=\frac{27,5}{3,15}=8,73<\frac{54}{\sqrt{F_{y}}}=10,8
$$

Como 8,73 é menor que 10,8 admitimos a mesa como sendo compacta.

Verificação do apoio lateral

Para considerarmos que a viga será apoiada lateralmente, ela precisa, ela precisa atender à duas condições conforme citadas 2.2.1.7.2.

$$
\begin{aligned}
& \frac{63 \times 25}{\sqrt{25}}=693 \mathrm{~cm} \\
& \frac{14060}{\left(\frac{d}{A_{f}}\right) \times \sqrt{F y}}=5128 \mathrm{~cm}
\end{aligned}
$$

Como nenhuma os dois valores foram maiores que Lb $(2400 \mathrm{~cm})$, consideramos a viga sem apoio lateral completo.

Cálculo da tensão admissivel à flexão

Visto que a estrutura é compacta sem apoio lateral, sua tensão admissível dependerá do resultado do comprimento do apoio lateral sobre o raio de giração:

$$
r_{t}=\sqrt{\frac{I_{y}}{2 \times\left(A_{f}+\left(\frac{A_{w}}{6}\right)\right)}}=\sqrt{\frac{87400}{2 \times\left(173,25+\left(\frac{168,58}{6}\right)\right)}}=14,7325 \mathrm{~cm}
$$


Portanto:

$$
\frac{L_{b}}{r_{t}}=\frac{2400}{14,7325}=162,90
$$

Desta forma:

$$
\frac{L_{b}}{r_{t}}>\sqrt{\frac{\left(358580 \times C_{b}\right)}{F_{y}}} \Rightarrow \sqrt{\frac{(358580 \times 1,0)}{25}}
$$$$
\Rightarrow \quad 162,90>120
$$

Como a verificação é verdadeira, as duas equações usadas para definição da tensão admissível são mostradas abaixo, sendo escolhido entre elas o maior valor:

$$
\begin{aligned}
& F b^{\prime}{ }_{x}=\frac{119520 \times C_{b}}{\left(\frac{L_{b}}{r_{t}}\right)^{2}} \leq 0,60 \times F_{y} \cong 4,5 \\
& \mathrm{E}: \\
& F b^{\prime \prime}{ }_{x}=\frac{8430 \times C_{b}}{\frac{L_{b} \times d}{A_{f}}} \leq 0,60 \times F_{y} \quad \cong \quad 6,41
\end{aligned}
$$

Como a tensão máxima admissível é $60 \%$ da tensão de escoamento, fazemos mais um teste:

$$
F b^{\prime \prime}{ }_{\text {max }}=25 \times 0,6 \cong 15 \mathrm{kN} / \mathrm{cm}^{2}
$$

Como $F b^{\prime \prime}{ }_{x}<F b^{\prime \prime}{ }_{\text {max }}$, utilizamos como tensão admissível o valor de $F b^{\prime \prime}{ }_{x}=6,41 \mathrm{kN} / \mathrm{m}^{2}$. 
Por fim, multiplicamos o maior valor de Fb permitido pelo valor de Wx obtido diretamente da tabela do fornecedor:

$$
\begin{aligned}
& F b_{x}=\frac{M_{x}}{W_{x}} \leq F b_{x} \times Q \times Q_{e} \\
& M_{x}=6,41 \times 17720 \\
& M_{x}=F b_{x} \times W_{x} \cong 113.591 \mathrm{kN} . \mathrm{cm}
\end{aligned}
$$

Para transformar em carga distribuída $(p)$, utilizamos a equação:

$$
M_{x}=\frac{p \times L^{2}}{8} \Rightarrow p=\frac{M_{x} \times 8}{L^{2}} \Rightarrow p=\frac{113591 \times 8}{2400^{2}}=0,1578 \mathrm{kN} / \mathrm{cm} \text { ou } 15,78 \mathrm{kN} /
$$

$\mathrm{m}$

Como PT $<p(12,967<15,78)$, o perfil utilizado suporta a demanda de carga sofrida, e pode ser utilizado. Apesar do valor estar próximo da máxima carga por metro possível, vale lembrar que dentro das fórmulas de dimensionamento já foram realizadas convenções para maximizar o fator de segurança, especialmente a de que todos os cenários foram considerados com o pior caso.

\section{CONCLUSÃO}

O projeto de uma passarela, mesmo que de pequeno porte, tem consequências muito impactantes em sua sociedade, pois diminui o fluxo de pedestres nas demais alternativas, além de fornecer um trajeto mais rápido para o cidadão que realiza seus percursos caminhando. Com o desenvolvimento deste projeto, pudemos concluir que existe uma demanda de novas alternativas de rotas para os moradores dos bairros Krüger, Santa Catarina e DER, para que possam atravessar o Rio do Peixe de forma segura e em um menor percurso, já que suas únicas travessias se encontram a uma distância superior a 1 km.

Dentre outras considerações, o crescimento populacional do globo nos indica diversos fatores nos quais precisamos estar preparados para evitar um caos 
nas logísticas de transporte, e um deles é ordenar o tráfego (neste caso, de pedestres) de forma a garantir um fluxo ininterrupto, seguro e acessível, para as crescentes quantidades de demanda. Esse fluxo, embora muitas vezes desconhecido pelo projetista, altera constantemente a necessidade de novas passarelas, para isso é necessário um monitoramento das travessias atuais. O engenheiro civil, como também é cidadão, precisa estar atento à essas necessidades para contribuir com o crescimento e organização de sua sociedade.

O dimensionamento de uma passarela de estrutura metálica, bem como qualquer estrutura de concreto armado, precisa estar precisamente dimensionada e com a adoção de diversos fatores de segurança para evitar catástrofes. Além disso, por vezes são necessárias adaptações não previstas, como foi o uso de um perfil soldado ao invés de um laminado para suportar os esforços solicitantes nas vigas. Fica à critério do engenheiro qual a solução mais viável à se utilizar, tendo em vista principalmente o funcionamento estrutural da edificação e seu bom aspecto arquitetônico.

\section{REFERÊNCIAS}

ASSOCIAÇÃO BRASILEIRA DE NORMAS TÉCNICAS. NBR 6120: Cargas para o cálculo de estruturas de edificações. Rio de Janeiro, 1980.

ASSOCIAÇÃO BRASILEIRA DE NORMAS TÉCNICAS. NBR 6123: Forças devidas ao vento em edificações. Rio de Janeiro, 1988.

ASSOCIAÇÃO BRASILEIRA DE NORMAS TÉCNICAS. NBR 7188: Carga móvel em ponte rodoviária e passarela de pedestre. Rio de Janeiro, 1984.

ASSOCIAÇÃO BRASILEIRA DE NORMAS TÉCNICAS. NBR 8800: Projeto de estruturas de aço e de estruturas mistas de aço e concreto de edifícios. Rio de Janeiro, 2008.

ASSOCIAÇÃO BRASILEIRA DE NORMAS TÉCNICAS. NBR 9763: Aços para perfis laminados, chapas grossas e barras, usados em estruturas fixas. Rio de Janeiro, 1987.

CBCA. Aços estruturais. Disponível em: <http://www.cbcaacobrasil.org.br/site/construcao-em-aco-acos-estruturais.php>. Acesso em: 08 nov. 2016. 
FIALHO, Antônio de Pádua Felga. Passarelas urbanas em estrutura de aço. Ouro Preto, 2004.

GASPAR, Ricardo. Estruturas metálicas: Estruturas metálicas, de madeiras especiais. São Paulo, SP, 2008.

NETO, Augusto Cantusio. Estruturas metálicas - Notas de aula. Pontifícia Universidade Católica - Centro de Ciências Exatas Ambientais e de Tecnologias PUC / CEATEC. Campinas, 2007.

PFEIL, W. Concreto armado: Dimensionamento. 2ed. Rio de Janeiro: Livros Técnicos e Científicos, 1983.

PROFISSIONAL DO AÇO. Aço ASTM-A36 e sua utilização. Disponível em: $<$ https://www.profissionaldoaco.com.br/destaques_in.asp?id_destaque=137>. Acesso em: 08 nov. 2016.

TRIMETAIS. O que é aço A36. Disponível em:

<http://trimetais.com.br/materiais/aco-a36/>. Acesso em: 08 nov. 2016.

QUADROS, Bibiana Cunha. Passarela em arco com tabuleiro inferior: Proposta de projeto para o campus central da Universidade Federal do Rio Grande do Sul. Porto Alegre, 2012. Disponível em:

<https://www.lume.ufrgs.br/bitstream/handle/10183/79760/000896680.pdf?sequ ence $=1>$. Acesso em: 20 set. 2016. 